\title{
Presbyastasis: a multifactorial cause of balance problems in the elderly
}

\section{Rogers MSc(Aud)}

To cite this article: C Rogers MSc(Aud) (2010) Presbyastasis: a multifactorial cause of balance problems in the elderly, South African Family Practice, 52:5, 431-434, DOI: 10.1080/20786204.2010.10874018

To link to this article: http://dx.doi.org/10.1080/20786204.2010.10874018

(c) 2010 SAAFP. Published by Medpharm.

曲 Published online: 15 Aug 2014.

Submit your article to this journal $\sqrt{3}$

!ll Article views: 412

Q

View related articles $\sqsubset$ 


\title{
Presbyastasis: a multifactorial cause of balance problems in the elderly
}

\author{
Rogers C, MSc(Aud) \\ Division of Communication Sciences and Disorders, School of Health and Rehabilitation Sciences, \\ Faculty of Health Sciences, University of Cape Town \\ Correspondence to: Christine Rogers, e-mail address: Christine.Rogers@uct.ac.za \\ Keywords: dizziness; elderly; presbyastasis; vestibular hypofunction
}

\begin{abstract}
Presbyastasis is the result of age-related physiological changes in the three sensory systems and their central connections that contribute to balance. In all likelihood, presbyastasis is a complex condition involving many intertwined systems rather than a lesion within the vestibular system only, thus evaluation and management need to be holistic. Balance problems can have detrimental consequences and are associated with falls, loss of quality of life and psychological sequelae such as anxiety, depression and panic. Healthcare practitioners need increased awareness of the pathophysiology of presbyastasis and its possible impact.
\end{abstract}

(P) Peer reviewed. (Submitted: 2010-01-11, Accepted: 2010-05-14). ๑ SAAFP

SA Fam Pract 2010;52(5):431-434

\section{Introduction}

One of the first challenges in assessing and managing the dizzy patient is to ascertain the exact nature of the complaint. A frequently used typology forming the basis of dizziness definition is: vertigo, presyncope, dysequilibrium, and other dizziness. ${ }^{1}$ The term "vertigo" has different meanings for both patients and healthcare practitioners, however a generally agreed upon definition is that vertigo is a definite illusion of movement, which is usually, but not always, rotatory. ${ }^{2}$ A clear description of vertigo from a patient significantly predicts a unilateral peripheral vestibular disorder, especially if it is associated with nausea and vomiting. ${ }^{3,4}$ Presyncope is the feeling of impending fainting and is often associated with hyperventilation or changes of vascular supply to the brain. Dysequilibrium is a sensation of imbalance (often reported to be "in the legs") when standing or walking and has a range of causes, from vestibulospinal problems to psychological diagnoses. "Other dizziness" includes descriptions of floating, swimming or less defined sensations. Numerous clinicians rely on this typology to drive their clinical reasoning and thus further investigations, management and referrals. However, slotting symptoms into strict categories can be confining in the case of multisensory impairment, as is to be expected in the elderly. Many patients, in particular the aged, cannot place their dizziness in just one category; and about half of older people have two or more subtypes of dizziness. ${ }^{1}$ The presence of multiple, sometimes nebulous, symptoms and possible aetiologies adds to the challenges that the dizzy patient brings to the consultation.

When the balance system is functioning optimally, it is able to correct for changes to the centre of gravity, provide information about the position of the body and finally, via the vestibulo-ocular reflex, ensure clear visual fields when either the head or the head and the environment are moving. Postural control must be preserved at rest (static balance) and moving (dynamic balance) in order to remain safe and avoid falls. Maintenance of balance is a complex function. Input is provided from three key receptor groups at the periphery of the nervous system: visual, proprioceptive and vestibular. Analysis of the incoming signals and responses to changes which could threaten balance occur in the dedicated integration areas of the brainstem and cerebellum. Commands to the eyes and musculoskeletal system from the central integrators deliver output responses to stabilise vision and postural control. A breakdown or mismatch within or between these three key senses, the processing areas in the brain or the output mechanisms can result in problems with maintenance of balance. The symptomatic complaints may be communicated along a continuum of dizziness, dysequilibrium or vertigo. ${ }^{5}$

With ageing, degenerative changes in the physiological function of all receptor and integration areas, combined with cardiovascular and other diseases, plus polypharmacy, can lead to debilitating dizziness which may affect every aspect of the patient's life. The family practitioner may be asked to make a diagnosis, recommend treatment strategies and weigh the needs of the patient and the family when the patient's independence is compromised. While the "greying" of the population is perceived to be a problem in the developed world, data suggest that, in spite of the HIV/AIDS pandemic, and high infant, child and adult mortality, the absolute number of older individuals in Africa is expected to increase from 47.4 million in 2005 to 193 million in 2050 . $^{6}$ Thus it is reasonable to suggest that 
balance problems related to age could become an emerging problem in developing countries, and therefore clinicians need to take heed. It is likely that in Africa, management of presbyastasis could be compounded by issues such as a limited focus on geriatric concerns in training, a lack of healthcare practitioners with expertise in balance rehabilitation and fall prevention, and stretched resources to manage the consequences of falls on patients and their families, for example, subsequent institutionalisation. ${ }^{6}$ The focus of this article is to describe the age-related changes in balance and their impact in an elderly population.

\section{Physiological and pathological changes with ageing}

Decline in balance and postural control may be due to either specific pathology in the contributing senses and integration centres, or the general progressive loss of function expected with ageing. The three main inputs into postural control will be discussed, followed by the implications of vestibular deficits.

\section{Visual}

Visual inputs allow for sensing orientation of the body in space as well as imprinting a visual picture of the environment on the brain, which in turn allows for navigation around potential hazards. Visual difficulties may result in potential mismatches of distance and inaccurate processing of spatial information, making it more challenging to negotiate obstacles and maintain postural control. For example, impaired depth perception and contrast sensitivity may result in trips over steps, curbs, or broken pavements. ${ }^{7}$ Multifocal spectacles are particularly problematic in patients with balance problems. Vision deteriorates from the age of 50 and the effects of pathology such as cataracts or diabetic changes may be superimposed on this. Furthermore, the ability to respond to changes in visual input also declines as a function of age. Young individuals are able to tolerate alterations in vision and still maintain postural control; however, older people take longer to respond to, or recover from, visual disruptions, ${ }^{7}$ explaining why patients often complain of dizziness and dysequilibrium after cataract surgery - a phenomenon that may be mistakenly attributed to intra operative events.

\section{Proprioceptive}

Proprioceptive inputs include information from the lower limbs, which is the most important contributor during standing balance. During walking, proprioception provides the required information to execute each step and to ensure optimal foot placement. Increasing age leads to changes in the quality and quantity of proprioceptive inputs which could lead to postural instability. Impairment in proprioceptive information may be the result of a loss of sensation caused by disease, for example diabetic peripheral neuropathy. Changes also result from a decline in muscle mass which begins from the $6^{\text {th }}$ decade and affects leg strength and power. In addition, speed and organisation of muscle force slows down. The combination of these factors may cause difficulty in stepping or correcting from tripping, thus increasing the risk of falling. Degeneration of the cervical spine is common in old age and in turn this may disturb postural control and may contribute to falls. ${ }^{7}$

\section{Vestibular}

The sensory epithelium in the labyrinthine end organs detects linear and angular acceleration. Similar to presbyacusis (agerelated hearing loss) there are anatomical and physiological changes in the vestibular hair cells, especially those found in the ampulla; collectively termed presbyastasis. Echoing presbyacusis, degenerative changes are not only confined to the end organ and the rate of loss in the vestibular nuclei occurs at a rate of $3 \%$ per decade after the age of 40.5 . Schuknecht examined temporal bones to explore the effect of ageing in the ear and at the same time as describing the different types of presbyacusis, he documented four types of presbyastasis: ampullary, macular, vestibular ataxia and cupulolithiasis. Interestingly, his description of cupulolithiasis laid the foundation for much of the later work on benign paroxysmal positional vertigo (BPPV) and its treatment with particle repositioning manoeuvres. It is now well established that the chances of developing BPPV definitely increase with ageing - by the age of 70 one third of all people will have had at least one occurrence of BPPV. ${ }^{8}$ It is reasonable to suggest that, similar to presbyacusis in which a symmetrical sensorineural hearing loss would develop, the physiological changes in the vestibular system would be symmetrical. This is important in terms of the description of the patient's presenting complaint, which is more likely to be dysequilibrium rather than vertigo, which is usually associated with an acute unilateral loss of vestibular tone. Hence, the term presbyastasis is preferable to the alternative proposals of presbyvertigo or presbyataxia. ${ }^{8}$

One of the most important functions of the vestibuloocular reflex arc is to match head movement with equal and opposite eye movement, termed gain. The gain of the vestibular system, plus the ability to generate redirective eye movements known as saccades and visually track objects (smooth pursuit) all decline with age. The result of reduced gain is that rapid head movements, for example the head movements when driving, are not matched by the appropriate degree and speed of eye movement. However, because of the plasticity of the brain and the remarkable ability of the vestibular system to recover from a loss of input, the cerebellum is able to compensate for the reduced sensitivity. Nevertheless, over time, the combination of reduced cues from the endorgan receptors and central inhibition reduces the range in which the balance system can respond. Disorders impacting on the quality and quantity of vestibular inputs may add to the effect of age. These include BPPV, which can also present with marked gait imbalance as well as positional vertigo; drug toxicity which is not confined only to vestibulotoxics, migraine and cerebellar ataxia. Symptoms may range from dizziness and dysequilibrium to fully blown vertigo accompanied by nausea and vomiting depending on the site and severity of the lesion, and if it is uni- or bilateral. ${ }^{7,5}$ 


\section{Changes in gait, falls and vestibular lesions}

Changes in gait that are associated with vestibular hypofunction include postural instability, broad-based gait, staggering, and unsteadiness on turns, all of which can increase the risk of falls. However, in some patients these changes may only be apparent when visual or proprioceptive challenges are added. This could partially explain why the elderly are more likely to fall at night when getting up to go to the bathroom. Results of studies which have explored the link between vestibular deficits and falls in the elderly are not conclusive. There are several influencing factors. First, patients may be aware of their difficulty and employ a variety of strategies to prevent falls, such as becoming more cautious when transferring or walking; attempting to minimise risk and using correction strategies or even assistive devices such as canes. Second, the issue of vestibular compensation may permit some functional recovery even though the vestibular changes are permanent. Third, questionnaires, bedside tests and screening assessments used in epidemiological studies to measure vestibular function may have inadequate sensitivity and specificity to detect impairment in vestibular function. ${ }^{7}$ Finally, specialised equipment capable of establishing vestibular impairments, such as videonystagmography and rotary chair, is expensive and a considerable amount of technical expertise is required to administer and interpret the results. ${ }^{9}$ The last factor is especially pertinent in developing countries. In spite of the potential pitfalls when exploring vestibular function and falls, there has been some new evidence which is most compelling. Jacobson et al's research used quantitative tests of vestibular function to evaluate patients referred for the assessment of falls risk. The majority of participants (73\%) were discovered to have vestibular deficits. ${ }^{9}$ Other authors have found that bilateral impaired vestibular function (as would be expected in presbyastasis) increases risk of falls and injury in patients over 65 years. ${ }^{10}$ Furthermore, in a large study, subjects who had measureable vestibular dysfunction and selfreported dizziness had a risk of falling which was increased twelvefold. The same study suggested that subjects who were asymptomatic but with evidence of vestibular fallout also had significantly increased odds of falling. ${ }^{11}$ While definitive evidence may have been lacking until recently, it would seem fair to suggest that links between vestibular deficits and fall risk have now been established.

To summarise, it is probable that balance declines in the elderly due to age-related degeneration, potentially aggravated by infections and injuries which can affect the sensory, motor and adaptive components of balance function. Mild changes in any one system may not lead to significant disturbances of control, but if there is a combination of these factors then there may be severe balance deficits, for example diabetes may cause retinopathy and peripheral neuropathy, causing fallout in two of the three key inputs. Furthermore, it is thought that the time taken to transmit stimuli, integrate them centrally and initiate movements in response is slower in the elderly.
Even simple reactions can take $25 \%$ longer than in a young person. In managing complex tasks, for example, walking and talking, reaction time is prolonged. It is most likely that presbyastasis is a generalised problem involving many intertwined systems rather than a lesion within the vestibular system only. ${ }^{8}$ General issues such as orthostatic hypotension, polypharmacy, dehydration, anaemia and even stress may create a tipping point which results in dizziness, lightheadedness or presyncope. ${ }^{7,12}$

\section{Evaluation and management of presbyastasis}

Dizziness is a common complaint in general practice and is associated with negative consequences such as falls, decreased quality of life and psychological sequelae such as anxiety, depression and panic. ${ }^{13}$ In spite of the high prevalence of the problem, there are no guidelines that address dizziness presentations in a wide sense. ${ }^{14}$ However, there are specialised guidelines for the diagnosis of certain vestibular disorders such as Ménière's Disease and BPPV. Two guidelines suggest that specialised vestibular tests have clinical utility, but lack information about sensitivity, specificity and likelihood ratios. ${ }^{14}$ Furthermore, it could be argued that specialised testing may often fail to elucidate symptoms, demonstrate pathology or assess compensation. It is certainly expensive and not readily available in developing countries. However, the ability to categorise symptoms, perform the appropriate examination, and offer an explanation and treatment options is within the realm of the family practitioner. ${ }^{3}$ In spite of this, it would seem as if there are barriers for many clinicians who deal with dizzy patients. These include a lack of confidence when evaluating a dizzy patient, general dislike for dizzy patients (often due to the broad differentials), lack of knowledge in the area and time constraints. ${ }^{15}$

A Delphi procedure was recently conducted to obtain consensus on a diagnostic protocol for dizziness in elderly patients in general practice. ${ }^{16}$ As is echoed elsewhere in the literature, a crucial first step is to obtain symptom definition in the case history. While most $(69 \%)$ of the clinicians surveyed by Polensek et al routinely used this information to identify the cause of dizziness, it was variable across disciplines with $84 \%$ of audiologists obtaining a description of the dizziness and only $33 \%$ of geriatricians doing so..$^{15}$ It should also be noted that, as well as defining the symptom type, further questions should deal with information about the timing, duration and associated symptoms of the attack. ${ }^{17}$ The rest of the history in the protocol is focused on the use of medications, alcohol and general medical details. The suggested physical examination includes assessment of the locomotor, neurological and cardiovascular systems. Of particular importance is the assessment of orthostatic hypotension, which is prevalent in the older population, with up to $30 \%$ of patients over the age of 75 meeting the diagnostic criteria. ${ }^{18}$ While age is associated with reduced baroreflex responsiveness, another common cause may be medication..$^{19,20}$ Of interest is that the protocol of Maarsingh et al includes otoscopy and the use of the Dix-Hallpike 
manoeuvre to assess the possibility of BPPV. The inclusion of a recommendation to routinely test for the presence of BPPV is important as BPPV is common in the elderly. Disappointingly, there have been several studies which have suggested that the Dix-Hallpike is not performed regularly, which will lead to misdiagnosis and inappropriate treatment of a condition which is easily, inexpensively and successfully treated with repositioning procedures. ${ }^{21,17,22,23} \mathrm{~A}$ potential weakness in the protocol is that no tests of vestibulo-ocular reflexes are suggested. While the latter tests are routine in the neuro-otological setting, they can be accomplished without the use of specialised equipment and would therefore lend themselves to use in family practice. All dizzy patients could possibly benefit from simple vestibular screening tests such as head shake, head thrust and hyperventilation. ${ }^{21,22}$ The tests are very helpful in detecting asymmetrical vestibular tone (which would support complaints of vertigo) or a component of anxiety, respectively. Informal evaluation of gait can be revealing, especially if the task is made more complex by requiring the patient to make head turns or talk when walking. Thus, a detailed case history, a general physical examination and some targeted tests could lead to diagnosis in the vast majority of patients with no need for expensive investigations such as MRI.

A discussion of the medical management of conditions such as orthostatic hypotension and polypharmacy is beyond the scope of this article. Problems within each of the sensory systems or central integrators should be isolated and treated if possible. However, it should be emphasised that vestibular sedatives are rarely effective for anything other than acute vertigo with nausea and vomiting and should never be used in the long term, when they are thought to delay or even prevent recovery. Research has shown that such sedatives may continue to be prescribed in primary care for months or even years. ${ }^{17}$ Whether the diagnosis is of presbyastasis or a more specific form of vestibular hypofunction such as BPPV, one of the biggest breakthroughs in treatment of vestibular impairments in the last twenty years has been the success of vestibular rehabilitation therapy (VRT). VRT is physiotherapy which drives compensatory changes in the brain, enhances visual stability and postural control, and finally is capable of reducing symptoms of dizziness and vertigo. ${ }^{13}$ It has been successfully applied in both specialist and primary care settings. Commonly it is a service provided by physiotherapists, although specially trained audiologists may also conduct such programmes. VRT has been shown to reduce symptoms of dizziness by as much as $85 \%$, and BPPV treatment with canalith repositioning procedures (which can be performed by physiotherapists, audiologists and medical practitioners) is effective in up to $97 \%$ of cases when performed correctly. ${ }^{21}$ However, referrals for VRT are frequently underutilised, ${ }^{21}$ suggesting that patients may continue to have expensive, inappropriate investigation and treatment while their quality of life continues to decline.

In summary, a combination of age-related degeneration in all three systems which contribute to postural control, and possible pathology in these or other systems, leads to presbyastasis or dysequilibrium of ageing. In addition to the physiological decline, elderly patients may have multiple medical problems which can impact on balance. Symptoms of dizziness, dysequilibrium and vertigo are usually episodic in the elderly but are disruptive to the activities of daily living, may have a profound effect on the quality of life and increase the risk of falling. Vigilance of the family practitioner and appropriate referrals, where necessary, may lead to better management and reduce morbidity and mortality.

\section{References}

1. Sloane P, Coeytaux R, Beck R, Dallara J. Dizziness: State of the Science. Ann Intern Med 2001;134 (9) Part 2:823-832.

2. Blakley B, Goebel, J. Meaning of the word Vertigo. Otolaryngol HeadNeck Surg 2001;125 (3):147-150.

3. Hanley K, O'Dowd T, Considine N. A systematic review of vertigo in primary care. Br J Gen Pract 2001;51 (469):666-671.

4. Chan Y. Differential diagnosis of dizziness. Curr Opin Otolaryngol Head Neck Surg 2009;17 (3):200-203.

5. Matsumura B, Ambrose A. Balance in the Elderly. Clin Geriatr Med 2006;22 (2):395-412.

6. Kulula SZ. A WHO report on falls among older persons. Prevention of falls in older persons: Africa case study. http://www.who.int/ageing/ projects/AFRO.pdf (Accessed 8 May 2009).

7. Sturnieks D, St George R, Lord S. Balance Disorders in the Elderly. Clin Neurophysiol 2008;38 (6):467-478

8. Belal A, Glorig A. Dysequilibrium of Ageing (Presbyastasis). J Laryngol Otol 1986;100 9):1037-1041.

9. Jacobson G, Mc Caslin D, Grantham S, Piker E. Significant vestibular system impairment is common in a cohort of elderly patients referred for assessment of falls risk. J Am Acad Audiol 2008;19(10):799-807.

10. Herdman $S$, Blatt $P$, Schubert $M$, Tusa R. Falls in patients with vestibular deficits. Am J Otolaryngol 2000;21 (6):847-851.

11. Agrawal Y, Carey J, Della Santina C, Schubert M, Minor L. Disorders of Balance and Vestibular Function in US Adults. Arch Intern Med 2009;169(10):938-944.

12. Katsarkas A. Dizziness in aging: the clinical experience. Geriatrics 2008;63(11):18-20.

13. Mira E. Improving the quality of life in patients with vestibular disorders: the role of medical treatments and physical rehabilitation. Int J Clin Pract 2007;62 (1):109-114.

14. Kerber K, Fendrick A. The evidence base for the evaluation and management of dizziness. J Eval Clin Pract 2010;16 (1):186-191.

15. Polensek S, Tusa R, Sterk $C$. The challenges of managing vestibular disorders: a qualitative study of clinicians' experiences associated with low referral rates for vestibular rehabilitation. Int $\mathrm{J}$ Clin Pract 2009;63(11):1604-1612.

16. Maarsingh $O$, Dros $J$, van Weert $H$, Schellevis $F$, Bindels $P$, van der Horst $H$. Development of a diagnostic protocol for dizziness in elderly patients in general practice: a Delphi procedure. BMC Fam Pract 2009;10(12) Accessed 8 April 2010 from www.biomedcentral. com/1471-2296/10/12

17. Newman-Toker D, Camargo L, Hsieh Y-H, Pelletier A, Edlow J. Disconnect between charted vestibular diagnosis and emergency department management decisions: a cross-sectional analysis from a nationally represented sample. Acad Emerg Med 2009;16 (10):970-977.

18. Gupta V, Lipsitz, L. Orthostatic hypotension in the elderly: diagnosis and treatment. Am J Med 2007, 120 (10):841-847.

19. Freeman R. Neurogenic orthostatic hypotension. New Engl J Med 2008;358 (6):615-624.

20. Bradley J, Davis K. Orthostatic hypotension. Am Fam Phys 2003,68(12):2393.

21. Polensek S, Sterk C, Tusa R. Screening for vestibular disorders: A study of clinicians' compliance with recommended practices. Med Sci Monit 2008;14(5):CR238-242.

22. Halmagyi M, Akdal G. Vertigo and imbalance. J Neurol Sci Turk 2005;22(2):123-141.

23. Von Brevern M, Radeke A, Lezius F, et al. Epidemiology of benign paroxysmal positional vertigo: a population based study. J Neurol Neurosurg Psych 2007;78 (7):710-715 